\title{
Effects of contrastive accents on children's discourse comprehension
}

\author{
Eun-Kyung Lee ${ }^{1}$ - Jesse Snedeker ${ }^{2}$
}

Published online: 27 May 2016

(C) Psychonomic Society, Inc. 2016

\begin{abstract}
What role do contrastive accents play in children's discourse comprehension? By 6 years of age, children use contrastive accents during online comprehension to predict upcoming referents (Ito et al., 2014; Sekerina \& Trueswell, 2012). But, at this age, children's performance on offline tasks of accent comprehension is poor (e.g., Wells et al., 2004). To examine whether the asymmetry could reflect a developmental stage in which the processing system uses contrastive accents to make local predictions, but fails to incorporate this information into discourse representations, we tested the effect of contrastive accents on children's memory of the content of a discourse. Five-year-olds heard 12 different stories consecutively, one after another, and the critical words were manipulated so that they were produced either with a contrastive $\mathrm{L}+\mathrm{H}^{*}$ accent or with a presentational $\mathrm{H}^{*}$ accent. We found that children remembered facts about the contrast set better when the target word had an appropriate contrastive accent earlier than when it had a presentational accent. The results show that by 5 years, children are able to use contrastive accents for encoding a discourse, as well as for making local predictions during online comprehension.
\end{abstract}

Eun-Kyung Lee

eunkyunglee@yonsei.ac.kr

1 Department of English Language and Literature, Yonsei University, 50 Yonsei-ro, Seodaemun-gu, Seoul 03722, Korea

2 Department of Psychology, Harvard University, Cambridge, MA 02138, USA
Keywords Language comprehension · Contrastive accents · Discoursre $\cdot$ Memory

\section{Introduction}

Pitch accents convey information about discourse structure by signaling information that is new or important, or contrasts with other information in the discourse (e.g., Bolinger, 1972; Brown, 1983; Chafe, 1976). Accented words are produced with increased duration and intensity, and greater pitch excursion. Consider (1).

\section{(1) John went to the MUSEUM.}

In (1), the presence of a pitch accent (marked by capital letters) on "museum" signals that "museum" is new information, or leads to an interpretation that John went to the museum, not to some other place. Pitch accents are used to predict upcoming referents during online processing (Arnold, 2008; Dahan, Tanenhaus, \& Chambers, 2002; Ito \& Speer, 2008; Weber, Braun, \& Crocker, 2006; Watson, Tanenhaus, \& Gunlogson, 2008). They also have long-term effects in adult language comprehension, modulating memory for the accented information (Fraundorf, Watson, \& Benjamin, 2010, 2012; Sanford, Sanford, Molle, \& Emmott, 2006), much like other linguistic devices for marking focus (e.g., Birch \& Garnsey, 1995; Fraundorf, Benjamin, \& Watson, 2013; Sanford et al., 2006; Sturt, Sanford, Steward, \& Dawydiak, 2004). For example, Fraundorf and colleagues (Fraundorf et al., 2010, 2012) find that facts about the discourse are better remembered when the critical word was produced with a contrastive $\mathrm{L}+\mathrm{H}^{*}$ accent earlier than with a pre- 
sentational $\mathrm{H}^{*}$ accent. ${ }^{1}$ However, relatively less is known about children's interpretation of contrastive accents. While it is clear that children are able to use contrastive accents for making local predictions (Ito, Bibyk, Wagner, \& Speer, 2014; Ito, Jincho, Minai, Yamane, \& Mazuka, 2012; Sekerina \& Trueswell, 2012), we do not know how these accents affect their subsequent representation of the discourse, or their ability to remember it. The present study explores this question in 5 -year-old children.

Studies of children's comprehension of contrastive accents have yielded mixed findings. Previous offline studies have failed to demonstrate children's sensitivity to contrastive accents (e.g., Cruttenden, 1985. Wells, Peppe, \& Goulandris, 2004). For example, in Cruttenden's (1985) study, 10 -year-olds were presented auditorily with utterances with a contrastive accent in varying locations, as shown in (2), and were asked to demonstrate their interpretation explicitly by selecting a matching picture.

\section{(2) a. John got FOUR oranges. b. John got four ORANGES.}

Children made a high proportion of judgment errors in picture selection. For example, the correct picture for sentence (2a) should be the one illustrating a boy with four oranges and a girl with two oranges. The result showed that only $65 \%$ of the children interpreted contrastive accents correctly. Wells et al. (2004) tested children from 5 to 13 years old on the interpretation of contrastive accents ("I wanted the CHOCOLATE and honey.” Which one didn't she get?). On this task, only 13 -year-olds showed accuracy above $75 \%$, suggesting that the ability to interpret contrastive accents continues to develop after the age of 5 years. Interpreting contrastive accents requires establishing a relevant contrast set. In order to interpret sentence ( $2 b$ ), children should be able to evoke alternatives of the focused entity 'oranges', not those of 'four'.

Why did the earlier studies fail to capture children's sensitivity to contrastive accents in offline tasks? Ito et al. (2012) have attributed children's poor performance on these offline tasks to the lack of a supportive discourse context. The support for this claim comes from eye-tracking studies exploring the role of contrastive accents in predicting upcoming referents. For example, in Ito et al. 's (2014) study, 6- to 11-year-old

\footnotetext{
${ }^{1}$ The $\mathrm{L}+\mathrm{H}^{*}$ and $\mathrm{H}^{*}$ notations are based on the ToBI (Tones and Break Indices) coding scheme developed for the transcription of speech intonation and prosody (Beckman, \& Ayers, 1997). The two types of pitch accents are distinguished by the shape of tonal movement: The $\mathrm{H}^{*}$ accent is associated with a high tonal target on the stressed syllable, while the $\mathrm{L}+\mathrm{H}^{*}$ accent is associated with a steep rise from a low tonal target to a high tonal target on the stressed syllable. The $\mathrm{L}+\mathrm{H}^{*}$ accent has been argued to convey a contrastive meaning whereas the $\mathrm{H}^{*}$ accent signals new information (Pierrehumbert, \& Hirschberg, 1990).
}

children heard a pair of sentences each containing a color adjective followed by a head noun.

(3) a. Where is the pink cat?

b. Now, where is the GREEN cat/green cat/GREEN monkey/green monkey?

The accompanying visual display contained multiple pictured animals each appearing in various colors. Children's eye movements to the objects in the display were measured while the second instruction $(3 b)$ was heard. If children interpret contrastive accents in the ways that adults do, they should expect a referent that is contrastive in color with the previously mentioned referent (e.g., green cat), at the moment of accented color adjectives in (3b). Six- to 11-year-olds fixated at a referent that was distinct in color from the previously mentioned referent (e.g., green cat) more frequently when the adjective was produced with contrastive accents (e.g., GREEN) than when it was not (e.g., green), suggesting that children were able to infer the speakers' intention conveyed by contrastive accents. Crucially, this effect occurred regardless of whether the upcoming noun was the same as (e.g., cat) or different from (e.g., monkey) the previously mentioned noun, suggesting that children used contrastive accents during online comprehension to predict upcoming referents. Although there were age differences in how quickly the effect came about, the data suggest that the children's poor performance in previous offline studies was not due to the failure to fully acquire the discourse meaning of contrastive accents. By 6 years, children seem to have implicit knowledge of contrastive accents and use them to make local predictions.

These findings are certainly consistent with the possibility that children understand contrastive accents but cannot make the right inferences in the absence of a sufficient discourse context. However, this is not the only explanation. Alternatively, the failures to find offline effects of contrastive accents could reflect that contrastive accents have only a fleeting effect in children: Children are sensitive to the contrastive accent but its effect does not persist long after the sentence is over. Ito and colleague's findings leave open the question of whether children's sensitivity to contrastive accents has an enduring effect on discourse interpretation. While it is known that children are sensitive to contrastive accents in making local predictions during online comprehension, no prior work has explicitly tested whether contrastive accents influence children's ultimate interpretation when the discourse is specified.

Fraundorf et al. (2010) show that contrastive accents have a persisting effect in adults. In their study, participants listened to a series of short stories each containing two contrast sets as illustrated in (4) below. 
(4) Both the British and the French biologists had been searching Malaysia and Indonesia for the endangered monkeys

(5) Finally, the (British/French) spotted one of the monkeys in (Malaysia/Indonesia) and planted a radio tag on it.

The stories continued with facts about one of the members in each contrast set as in (5). Pitch accenting on the critical word was manipulated so that it was produced either with a contrastive $\mathrm{L}+\mathrm{H}^{*}$ accent or with a presentational $\mathrm{H}^{*}$ accent. Recognition memory for facts about each critical item was tested (e.g., The British spotted the endangered monkey and tagged it.). The results showed that adults remembered facts about the critical word better when it had received an $\mathrm{L}+\mathrm{H}^{*}$ accent earlier than when it had received an $\mathrm{H}^{*}$ accent. While contrastive accents on the target word facilitated memory for facts about it, there was no detrimental effect of contrastive accents on the other critical word on memory for the target word.

The current study explores whether children are sensitive to contrastive accents in an offline task that provides fully specified discourse contexts. To investigate whether children's sensitivity to contrastive accents persists long after the sentence ends, we adapted the task from Fraundorf et al. (2010) for use with 5-year-olds. In the experiment reported below, children listened to a series of short stories each containing two contrast sets. Each contrast set contained two members that were explicitly mentioned in the stories. We tested whether contrastive accents affect children's memory for items in a contrast set. If children's failure to use contrastive accents in the earlier offline studies was due to fleeting features of their sensitivity to contrastive accents, there should be no effects of contrastive accents on children's memory of the focused information. If contrastive accents have a persisting effect in children, shaping their discourse representation, the contrastive accents should have a long-term consequence, affecting the memory of accented words. Testing this question does not tell us where in the processing stream the effect occurs. However, it allows us to assess the role contrastive accents play in children's ultimate comprehension.

Another goal is to test whether children's memory of the content of a discourse is impaired by a contrastive accent on some other entity in the discourse. Fraundorf et al. (2010) found no detrimental effects of contrastive accents for young adults. In their later study (Fraundorf et al. 2012), however, contrastive accents did impair memory for other information in a discourse in both older adults and young adults with low working memory capacity. Given that children's working memory performance is developmental in nature (Gathercole, Pickering, Ambridge, \& Wearing, 2004), it is possible that 5 -year-olds might also show detrimental effects of contrastive accents.

\section{Method}

\section{Participants}

Participants were 36 5-year-olds $(M=5.0$, range $=4: 5-5: 8 ; 21$ girls, 15 boys) recruited from the database of the Laboratory for Developmental Studies at Harvard University. All participants were native English speakers.

\section{Procedure}

Children were seated at a computer with an experimenter. In the instructions, children were told that they would be engaged in a memory task, and that they would listen to stories and be asked later about what happened in the stories. The experiment consisted of a study phase and a test phase with a delay period in between.

In the study phase, children heard 12 different stories such as (6) below consecutively, one after another.

(6) Annette went to a store to buy gifts for her brother and her father. She bought a scarf and a hat. She decided to give her brother the hat. He was very happy to get it.

Each story was presented over speakers. To help children link each story with a corresponding memory question, a picture illustrating either the story's setting (e.g., gift store, bakery), or the story's characters (e.g., children, family), was shown on the computer screen while each story was being played, and the same picture was presented in the test phase. However, the picture included no details related to the facts that were later tested.

In the delay period, children played arrow games on the computer: a Flanker task and a Simon task. In the Flanker task, children saw five fish appearing on the screen, and were asked to indicate the direction that the middle fish is facing by pressing either the right or left arrow. In the Simon task, children saw arrows appearing either on the left or right side of the screen. The arrows were pointing either to the left button ("W") or the right button ("P") on the keyboard. Children were asked to press a button that was being pointed to by arrows. This period lasted about $10 \mathrm{~min}$.

In the test phase, the memory test was performed in the same order in which the stories were presented in the study phase. Only one of the two contrast sets was tested for each story. To reduce a response bias, test probes had the form of an alternative question instead of a yes-no question as illustrated in (7) below. The order in which the target word was mentioned varied across items. Test probes were presented orally. The participants' verbal responses were recorded and later transcribed.

(7) Did Annette give her brother the hat or the scarf? 


\section{Materials}

Stimuli were 12 stories adapted from Fraundorf et al. (2010). Each story began with a context passage that established two contrast sets each consisting of two entities (e.g., brother/ father and scarf/hat) (8a). Each context passage was followed by a target sentence describing a fact about two critical entities, one each from each contrast set (8b).

(8) a. Context: Annette went to a store to buy gifts for her brother and her father. She bought a scarf and a hat.

b. Target: She decided to give her brother the hat. $\mathrm{He}$ was very happy to get it.

The target sentence was manipulated for which of the two nouns had a contrastive accent, which resulted in three different prosodic conditions as illustrated in (9).

(9) a. Baseline: She decided to give her brother the hat. b. Target word accented: She decided to give her brother the HAT.

c. Other word accented: She decided to give her BROTHER the hat.

In the baseline condition, both critical words were produced with an $\mathrm{H}^{*}$ accent. In the target word accented condition, the target word (e.g., hat) was produced with an $\mathrm{L}+\mathrm{H}^{*}$ accent, and the other critical word (e.g., brother) was produced with an $\mathrm{H}^{*}$ accent. In the other word accented condition, the target word received an $\mathrm{H}^{*}$ accent, and the other critical word had an $\mathrm{L}+\mathrm{H}^{*}$ accent. The $\mathrm{L}+\mathrm{H}^{*}$ accent was always followed by a prosodic boundary, which is typical for contrastive focus (Selkirk, 2002).

Stimuli were produced by a female native speaker of American English. To control for any unintended acoustic differences in the non-critical portion of the stories across conditions, a cross-splicing technique was employed. The critical words were cross-spliced so that they were embedded in an identical carrier passage across conditions. Recordings for the three conditions were used equally often as a carrier passage across stories.

As illustrated in Table 1, acoustic measures of duration, pitch, and intensity revealed that the critical words with a contrastive $\mathrm{L}+\mathrm{H}^{*}$ accent were produced with more acoustic prominence than their counterparts with a presentational $\mathrm{H}^{*}$ accent.

The speaker who produced the stories produced test probes with an $\mathrm{L}+\mathrm{H}^{*}$ accent on the first response choice, and an $\mathrm{H}^{*}$ accent on the second response choice (e.g., Did Annette give her brother the hat $t_{\mathrm{L}+\mathrm{H}^{*}}$ or the $\operatorname{scarf}_{\mathrm{H}^{*}}$ ?).

The 12 critical items were rotated through three prosody conditions across three lists. There were no filler stories. The order of the stimuli was pseudo-randomized and was identical in all lists. No two consecutive stimuli were presented in the same prosodic condition. Each child was assigned only one list. The position of test words was manipulated as betweenparticipants. Half of the children were tested on facts about the first contrast set (e.g., Did Annette give the hat to her brother or her father?). The other half were tested on facts about the second contrast set (e.g., Did Annette give her brother the hat or the scarf?).

\section{Results}

Response accuracy was coded as either 1 or 0 and was analyzed in a mixed logit model (Jaeger, 2008) with prosody (baseline vs. target word accented vs. other word accented), test word position (first critical word vs. second critical word), and their interaction as fixed effects. The model also included by-participant and by-item random intercepts, by-participant random slopes for prosody, and by-item random slopes for prosody, test word position, and their interaction as random effects. The maximal random effects model, which also included by-item random slopes for the prosody by test word position interaction, did not converge. The test word position variable was coded using mean contrast coding. The prosody variable was coded so that the effect of accent on target word and the effect of accent on other word were each assessed relative to the baseline condition.

Figure 1 illustrates the accuracy results in terms of the proportion of correct responses. Table 2 presents parameter estimates for the model.

Children remembered facts about the second critical word better than those about the first critical word, as revealed by a main effect of test word position (First: $82 \%$ vs. Second: $88 \%$; $\mathrm{z}=2.7, P<.01)$. While the mean in the other word accented condition was numerically higher than the baseline condition ( $85 \%$ vs. $81 \%)$, this difference was not significant $(\mathrm{z}=0.2, P>$ $.1)$, nor was there a significant interaction between accent on other word and test word position $(\mathrm{z}=-1.4, P>.1)$. There was also no main effect of accent on target word $(\mathrm{z}=1.1, P>.1)$. However, the interaction between accent on target word and test word position was significant $(\mathrm{z}=-2.8, P<.05)$, suggesting that mnemonic effects of contrastive accents varied by the position of test words.

Separate planned analyses revealed that there was a significant effect of $\mathrm{L}+\mathrm{H}^{*}$ on target word when facts about the first critical word were tested $(\beta=-2.3, \mathrm{SE}=0.7, \mathrm{z}=-3.3, P<$ $.01)$, but not when facts about the second critical word were tested $(\beta=0.2, \mathrm{SE}=0.6, \mathrm{z}=0.4, P>.1)$.

\section{Discussion}

This study explored the role of contrastive accents in children's discourse comprehension. We found that preschool children remembered facts about the critical item better when it had 
Table 1 Acoustic measures for critical words (means and standard errors)

\begin{tabular}{|c|c|c|c|c|c|c|}
\hline \multirow[t]{2}{*}{ Measure } & \multicolumn{3}{|c|}{ First critical word } & \multicolumn{3}{|c|}{ Second critical word } \\
\hline & $\mathrm{H}^{*}$ & $\mathrm{~L}+\mathrm{H}^{*}$ & $\mathrm{t}(11)$ & $\mathrm{H}^{*}$ & $\mathrm{~L}+\mathrm{H}^{*}$ & $\mathrm{t}(11)$ \\
\hline \multicolumn{7}{|l|}{ Stressed syllable } \\
\hline Duration (ms) & $213(21)$ & $564(77)$ & $5.9^{*}$ & $317(37)$ & $838(65)$ & $7.7^{*}$ \\
\hline Max Pitch (Hz) & $235(10)$ & $282(11)$ & $4.5^{*}$ & $211(4)$ & $299(30)$ & $2.8^{*}$ \\
\hline Pitch difference $(\mathrm{Hz})(\max -\min )$ & $40(11)$ & $94(11)$ & $3.8^{*}$ & $47(10)$ & $121(32)$ & $2.1^{* *}$ \\
\hline Mean Pitch $(\mathrm{Hz})$ & $211(5)$ & $234(9)$ & $3.0^{*}$ & $192(4)$ & $226(9)$ & $3.4^{*}$ \\
\hline Mean intensity $(\mathrm{dB})$ & $52(1)$ & $57(1)$ & $8.7^{*}$ & $49(1)$ & $54(1)$ & $5.4^{*}$ \\
\hline \multicolumn{7}{|l|}{ Entire word } \\
\hline Duration (ms) & $395(41)$ & $970(63)$ & $14.4^{*}$ & $404(37)$ & $957(45)$ & $14.1^{*}$ \\
\hline Max pitch (Hz) & $245(10)$ & $350(37)$ & $3.6^{*}$ & $211(4)$ & $300(30)$ & $2.8^{*}$ \\
\hline Pitch difference $(\mathrm{Hz})(\max -\min )$ & $59(11)$ & $193(42)$ & $4.3^{*}$ & $49(10)$ & $126(31)$ & $2.2^{* * *}$ \\
\hline Mean pitch $(\mathrm{Hz})$ & $209(5)$ & $219(3)$ & $2.4^{*}$ & $191(3)$ & $222(9)$ & $3.0^{*}$ \\
\hline Mean intensity $(\mathrm{dB})$ & $52(1)$ & $55(1)$ & $8.0^{*}$ & $48(1)$ & $53(1)$ & $7.6^{*}$ \\
\hline
\end{tabular}

been produced with an appropriate, contrastive $\mathrm{L}+\mathrm{H}^{*}$ accent earlier than with a presentational $\mathrm{H}^{*}$ accent. This pattern suggests that 5 -year-olds incorporated information conveyed by the contrastive accent into their ultimate interpretation of a discourse. While children's memory for the critical word was enhanced by the presence of a contrastive accent, it was not impaired by the presence of a contrastive accent on the other critical word. This same asymmetry has been found in adults (Fraundorf et al., 2010, but see Fraundorf et al., 2012). Thus, at both ages, the apparent effect of contrastive accents is to facilitate memory for information in focus, and not to impair memory for other information in the discourse.

Previous studies have shown that contrastive accents improve adults' memory for the focused entity by enhancing a representation of a contrast item rather than a representation of the focused entity itself (e.g., Braun \& Tagliapietra, 2010;
Fraundorf et al., 2010). The current experiment does not allow us to test the exact mechanism underlying the effect of contrastive accents on children's memory.

Nevertheless, these findings clearly allow us to draw new conclusions about the role of contrastive accents in children's comprehension. The results of Ito and colleagues $(2012,2014)$ demonstrate that children are sensitive to the contrastive accent as they are hearing it, and can make appropriate predictions about upcoming referents. The present findings demonstrate that this sensitivity to contrastive accents persists, shaping the child's memory for accented words long after the sentence is over. Thus, the effects of contrastive accents are not fleeting, momentary fluctuations in attention but rather reflect more enduring features of children's representation of the discourse. These effects could occur at multiple points in the processing stream: as a part of linguistic analysis, during
Fig. 1 Proportion of correct responses as a function of prosody and test word position (means and standard errors)

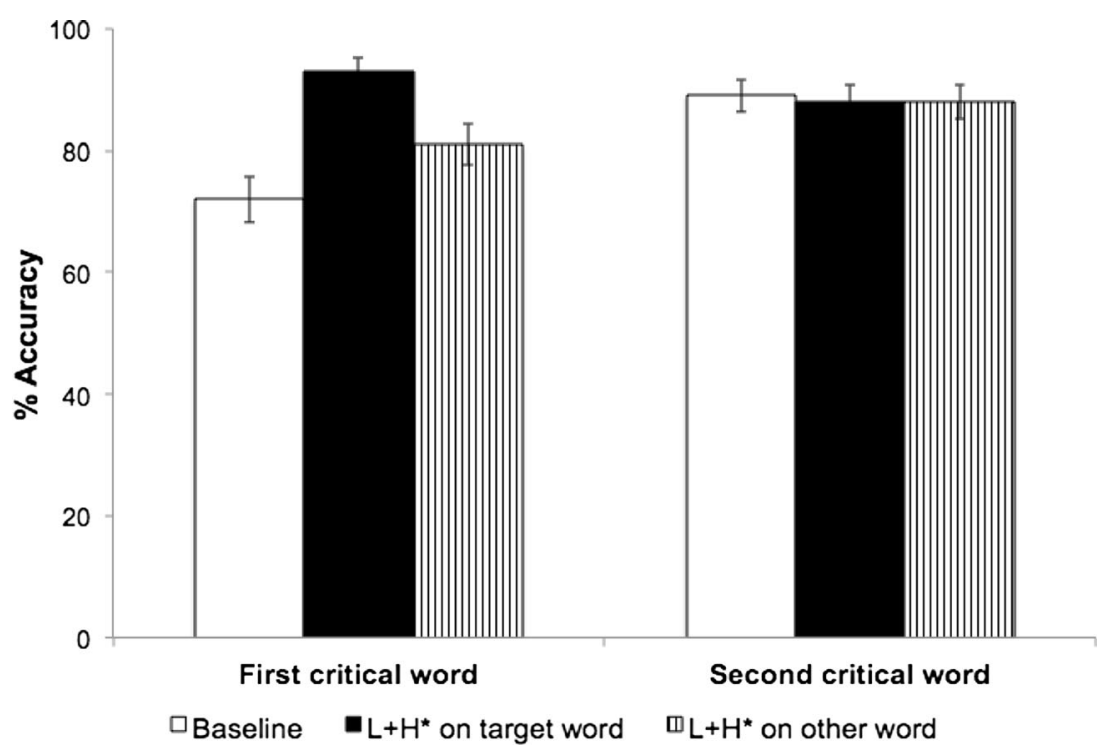


Table 2 Mixed logit models with prosody, test word position, and their interaction as fixed effects $(\mathrm{N}=432$; log-likelihood $=$ $-167.9)$

\begin{tabular}{lllll}
\hline & Estimate & SE & Z-value & $P$-value \\
\hline Fixed effects & & & & \\
(Intercept) & 2.0 & 0.4 & 5.0 & $<.01$ \\
$\mathrm{~L}+\mathrm{H}^{*}$ on target word & 0.5 & 0.4 & 1.1 & $>.1$ \\
$\mathrm{~L}+\mathrm{H}^{*}$ on other word & 0.1 & 0.4 & 0.2 & $>.1$ \\
Test word position & 1.7 & 0.6 & 2.7 & $<.01$ \\
$\mathrm{~L}+\mathrm{H}^{*}$ on target word $\times$ test word position & -2.2 & 0.8 & -2.8 & $<.01$ \\
$\mathrm{~L}+\mathrm{H}^{*}$ on other word $\times$ test word position & -1.0 & 0.7 & -1.4 & \\
& Variance & & & \\
Random effects & & & & \\
Participant (intercept) & 0.7 & & & \\
$\mathrm{~L}+\mathrm{H}^{*}$ on target word & 0.1 & & \\
$\mathrm{~L}+\mathrm{H}^{*}$ on other word & 0.0 & & \\
Item (intercept) & 0.8 & & \\
$\mathrm{~L}+\mathrm{H}^{*}$ on target word & 0.4 & & \\
$\mathrm{~L}+\mathrm{H}^{*}$ on other word & 0.3 & & \\
Test word position & 0.7 & & \\
\hline
\end{tabular}

memory encoding, or during retrieval. These alternatives have not all been cleanly teased apart in the adult studies, and they are not disentangled here. However, by demonstrating that the interpretive effects of contrastive accents persist in young children, we have taken one small but critical step toward understanding how this system develops.

Our findings show that the memory benefit of contrastive accents varied by the position of test words: only memory for facts about the first of the critical words benefited from contrastive accents. One possible explanation is that the absence of a mnemonic benefit of contrastive accents for second critical words may reflect the mechanism by which contrastive accents yield a boost to children's memory. Contrastive accents may affect children's memory by providing extra time for elaborating the representation of focused entities. In our stimuli, the second critical words appeared towards the end of the discourse, which means that there was less material to follow compared to the first critical words. Thus, children could have had sufficient time to encode the second critical words even in the non-contrastive accent condition, resulting in no boost to memory due to extra time provided by contrastive accents.

Another possible explanation is that given that test word position was manipulated as a between-participants factor, the asymmetry may reflect different participants. Previous work by Wells et al. (2004) demonstrated that child age was a critical factor that might influence children's sensitivity to contrastive accents in offline comprehension. In the current study, the two groups of children did not significantly differ from each other in age $(P>.1)$. Nevertheless, there still remains a possibility that the asymmetry may reflect differences in cognitive abilities. Future work is required to explicitly test these possibilities. Despite the lack of effects of contrastive accents for second critical words, we did find that the $\mathrm{L}+\mathrm{H}^{*}$ accent benefited children's memory for first critical words. The presence of a memory benefit of contrastive accents for first critical words suggests that contrastive accents do influence 5year-olds' memory representation of a discourse.

One might argue that the memory benefit of contrastive accents could be a purely lexical phenomenon. Perhaps the acoustically prominent words stayed more accessible because the words themselves were more active, not because they were integrated more effectively into the discourse. We had two stories in the set that happened to contain an identical contrast set, allowing us to test this directly. Those trials contained a contrast set whose members were "boys" and "girls". On one trial, the first critical word in the target sentence was "boys" (i.e., the correct answer to the memory question was "boys".) and on the other trial, it was "girls". If contrastive accents had a mnemonic effect because of their acoustic salience, we should have found a chance performance on these two trials because each individual child heard both "boys" and "girls" with either a contrastive accent or a presentational accent. When only these two items were included in the analysis, there was still an effect of contrastive accents on the target word ( $\mathrm{L}+\mathrm{H}^{*}: 92 \%$ vs. $\mathrm{H}^{*}: 67 \%$ ). This means that contrastive accents led children to choose between "boys" and "girls" correctly depending on the stories, demonstrating that the effect we found here reflects the effect of contrastive accents on the memory for the discourse and cannot be attributable simply to the effect of acoustic salience on lexical memory.

Our findings provide critical information about the development of the interface between prosody and discourse comprehension. The previous research introduced a puzzle: young children are able to rapidly use contrastive accents to make online predictions about upcoming referents (Ito et al., 2014) but they fail to use contrastive accents in offline judgment 
(Cruttenden, 1985; Wells et al., 2004). This discrepancy could reflect task demands: the previous offline tasks required participants to make inferences about an unknown context. Alternately, this discrepancy could have reflected fleeting features of children's sensitivity to contrastive accents. Our data answer this question. Children's later memory for a discourse is shaped by the presence of contrastive accents, indicating that their sensitivity to contrastive accents persists long after the sentence ends. This suggests that children's poor performance in interpreting contrastive accents in the prior offline studies was due to task demands. By 5 years, children are sensitive to contrastive accents, and this sensitivity not only shapes moment-to-moment language comprehension but also plays a role in their memory of what has been said.

Acknowledgments This work was supported by a grant from the Simons Foundation (SFARI 240290 to J.S.). We would like to thank Scott Fraundorf for providing us with example sound files and Michelle Gernon, Maria Massiani, Katie Lawlor, and Sabrina Ng for assistance with recording stimuli and running participants.

\section{References}

Arnold, J. E. (2008). THE BACON not the bacon: How children and adults understand accented and unaccented noun phrase. Cognition, 108, 69-99.

Beckman, M. E., \& Ayers, G. M. (1997). Guildlines for ToBI labelling, vers 3.0.. Columbus, $\mathrm{OH}$ : Ohio State University.

Birch, S. L., \& Garnsey, S. M. (1995). The effect of focus on memory for words in sentences. Journal of Memory and Language, 34, 232267.

Bolinger, D. (1972). Accent is predictable (if you're a mind-reader). Language, 48, 633-644.

Braun, B., \& Tagliapietra, L. (2010). The role of contrastive intonation contours in the retrieval of contextual alternatives. Language and Cognitive Processes, 25, 1024-1043.

Brown, G. (1983). Prosodic structure and the given/new distinction. In A. Cutler, \& R. Ladd (Eds.), Prosody: Models and measurements (pp. 67-77). New York: Springer.

Chafe, W. L. (1976). Givenness, contrastiveness, definiteness, subjects, topics, and point of view. In C. N. Li (Ed.), Subject and topic (pp. 25-56). New York: Academic Press.

Cruttenden, A. (1985). Intonation comprehension in ten-year-olds. Journal of Child Langauge, 12, 643-661.

Dahan, D., Tanenhaus, M. K., \& Chambers, C. G. (2002). Accent and reference resolution in spoken language comprehension. Journal of Memory and Language, 47, 292-314.
Fraundorf, S. H., Benjamin, A. S., \& Watson, D. G. (2013). What happened (and what did not): discourse constraints on encoding of plausible alternatives. Journal of Memory and Language, 69, 196-227.

Fraundorf, S. H., Watson, D. G., \& Benjamin, A. S. (2010). Recognition memory reveals just how CONTRASTIVE contrastive accenting really is. Journal of Memory and Language, 63, 367-386.

Fraundorf, S. H., Watson, D. G., \& Benjamin, A. S. (2012). The effects of age on the strategic use of pitch accents in memory for discourse: A processing-resource account. Psychology and Aging, 27, 88-98.

Gathercole, S. E., Pickering, S. J., Ambridge, B., \& Wearing, H. (2004). The structure of working memory from 4 to 15 years of age. Developmental Psychology, 40, 177-190.

Ito, K., Bibyk, S. A., Wagner, L., \& Speer, S. R. (2014). Interpretation of contrastive pitch accent in six- to eleven-year-old English-speaking children (and adults). Journal of Child Language, 41, 84-110.

Ito, K., Jincho, N., Minai, U., Yamane, N., \& Mazuka, R. (2012). Intonation facilitates contrast resolution: Evidence from Japanese adults and 6-year olds. Journal of Memory and Language, 66, 265-284.

Ito, K., \& Speer, S. R. (2008). Anticipatory effects of intonation: Eye movements during instructed visual search. Journal of Memory and Language, 58, 541-573.

Jaeger, T. F. (2008). Categorical data analysis: Away from ANOVAs (transformations or not) and towards logit mixed models. Journal of Memory and Language, 59, 434-446.

Pierrehumbert, J., \& Hirschberg, J. (1990). The meaning of intonational contours in the interpretation of discourse. In P. Cohen, J. Morgan, \& M. Pollack (Eds.), Intentions in communication (pp. 271-311). Cambridge: MIT Press.

Sanford, A. J. S., Sanford, A. J., Molle, J., \& Emmott, C. (2006). Shallow processing and attention capture in written and spoken discourse. Discourse Processes, 42, 109-130.

Sekerina, I. A., \& Trueswell, J. C. (2012). Interactive processing of contrastive expressions by Russian Children. First Language, 32, 63 87.

Selkirk, E. (2002). Contrastive FOCUS vs. presentational focus: prosodic evidence from right node raising in English. Proceedings of the 1 st International Conference on Speech Prosody, 643-646.

Sturt, P., Sanford, A. J., Steward, A. J., \& Dawydiak, E. (2004). Linguistic focus and good-enough representations: An application of the change-detection paradigm. Psychonomic Bulletin \& Review, 11, 882-888.

Watson, D. G., Tanenhaus, M. K., \& Gunlogson, C. A. (2008). Interpreting pitch accents in online comprehension: $\mathrm{H}^{*}$ vs. $\mathrm{L}+\mathrm{H}^{*}$. Cognitive Science, 32, 1232-1244.

Weber, A., Braun, B., \& Crocker, M. W. (2006). Finding referents in time: Eye-tracking evidence for the role of contrastive accents. Language and Speech, 49, 367-392.

Wells, B., Peppe, S., \& Goulandris, N. (2004). Intonation development from five to thirteen. Journal of Child Language, 31, 749-778. 